\title{
Condições de saúde bucal do idoso no Brasil: uma revisão de literatura
}

\author{
Oral health conditions of the elderly in Brazil: a literature review \\ Salud bucal del anciano en Brasil: una revisión de la literatura \\ Márcia Virgínia Gonçalves SALES ${ }^{1}$ \\ José de Alencar FERNANDES NETO² \\ Maria Helena Chaves de Vasconcelos CATÃO ${ }^{3}$ \\ ${ }^{1}$ Graduanda em Odontologia, Departamento de Odontologia, Universidade Estadual da Paraíba, UEPB, \\ 58429-500, Campina Grande - PB, Brasil \\ ${ }^{2}$ Mestrando do Programa de Pós-Graduação em Odontologia, Departamento de Odontologia, \\ Universidade Estadual da Paraíba, UEPB, 58429-500, Campina Grande - PB, Brasil \\ ${ }^{3}$ Professora Doutora do Programa de Pós-Graduação em Odontologia, Universidade Estadual da Paraíba, UEPB,
} 58429-500, Campina Grande-PB, Brasil

\begin{abstract}
Resumo
Introdução: A população brasileira vem sofrendo alterações demográficas com o passar dos anos. Esse aumento na expectativa de vida resultou no crescimento da população idosa do país, necessitando proporcionar às pessoas um envelhecimento com qualidade de vida. Objetivo: relatar as condições de saúde bucal do idoso brasileiro, enfatizando a qualidade de vida desse idoso, bem como o conhecimento dos cuidadores no que diz respeito à higiene e manutenção da saúde bucal dessa população. Material e método: Trata-se de um estudo bibliográfico, abrangendo a busca de artigos publicados nos últimos dois anos na Scientific Electronic Library Online (SciELO) sobre o tema "Condições de saúde bucal do idoso no Brasil". Utilizou-se como palavras-chave: "odontologia", "idoso" e "saúde bucal". Ao realizar a triagem desses artigos, utilizando-se os critérios de inclusão, têm-se para o estudo um total de doze artigos trabalhados, sendo sete (58,3\%) dos artigos publicados em 2014 e cinco (41,7\%) dos artigos publicados em 2015. Conclusão: Pode-se concluir com esse trabalho que existe a necessidade de tratamento odontológico voltado para essa população, devido às patologias oriundas do próprio envelhecimento.

Descritores: Envelhecimento; Odontologia Geriátrica; Saúde Bucal; Higiene Bucal.
\end{abstract}

\begin{abstract}
Introduction: The Brazilian population is undergoing demographic changes over the years. This increase in life expectancy has resulted in the increase of the elderly population of the country, which creates the need to providing people an aging process with quality of life. Objective: to report the oral health status of Brazilian elderly, emphasizing their quality of life, as well as the degree of knowledge of caregivers regarding the hygiene and maintenance of oral health of the elderly. Material and methods: This is a bibliographic study, including the search for articles published in the last two years in the Scientific Electronic Library Online (SciELO) on "Conditions of oral health of the elderly in Brazil". The following keywords were used: "Dentistry", "old" and "oral health". In performing the screening of these items, using the inclusion criteria, a total of twelve articles were colected, in which seven $(58,3 \%)$ of the articles were published in 2014 and five $(41,7 \%)$, in 2015 . Conclusion: It can be concluded from this work that there is a need for dental treatment facing this population due to diseases arising from aging itself.
\end{abstract}

Descriptors: Aging; Geriatric Dentistry; Oral Health; Oral Hygiene.

\begin{abstract}
Resumen
Introducción: La población brasileña está experimentando cambios demográficos en los últimos años. Este aumento de la esperanza de vida ha dado como resultado el crecimiento de la población anciana del país y una necesidad de ofrecer calidad de vida durante el envejecimiento. Objetivo: conocer el estado de salud bucal de adultos mayores brasileños, con énfasis en la calidad de vida de estos ancianos, así como el grado de conocimiento de los cuidadores en cuanto a la higiene y el mantenimiento de la salud oral de los ancianos. Material y método: Este es un estudio bibliográfico, incluyendo la búsqueda de artículos publicados en los últimos dos años en el Scientific Electronic Library Online (SciELO) con las siguientes palabras clave: "Odontología", "anciano" y "salud bucal". Al realizar la búsqueda de los estudios mediante los criterios de inclusión, se reunió un total de doce artículos, siete $(58,3 \%)$ de los artículos publicados en 2014 y cinco $(41,7 \%)$ de los artículos publicados en 2015. Conclusión: Se puede concluir a partir de este trabajo que hay una necesidad de tratamiento dental frente a esta población debido a las enfermedades derivadas de envejecimiento en sí mismo.

Descriptores: Envejecimiento; Odontología Geriátrica; Salud Bucal; Higiene Bucal.
\end{abstract}

\section{INTRODUÇÃO}

Desde o final do século XX o Brasil vem passando por uma alteração demográfica com um aumento acentuado da população acima dos $60 \operatorname{anos}^{1}$. Esse crescimento da expectativa de vida contribui para o aparecimento das doenças típicas da velhice, com envolvimento do sistema estomatognático, havendo, portanto, uma maior necessidade de tratamento odontológico voltado para essa população ${ }^{2}$.

Para o idoso, a qualidade de vida relaciona-se aos aspectos fisiológicos, patológicos, psicossociais, socioculturais, econômicos e ambientais. Essa qualidade de vida influencia diretamente no nível de sua saúde, quanto ao seu enfraquecimento funcional, morbidade e incapacidade ${ }^{3}$.
Com o envelhecimento, o organismo passa por algumas transformações, e a boca geralmente reflete, na velhice, as condições em que essas pessoas viveram. Tais condições se apresentam por alterações na cavidade bucal, como a cárie, a doença periodontal, o edentulismo, a redução do fluxo salivar e o acúmulo de biofilme dental ${ }^{4}$.

$\mathrm{O}$ presente trabalho objetiva relatar as condições de saúde bucal do idoso brasileiro; enfatizando a qualidade de vida desse idoso, bem como o grau de conhecimento dos cuidadores no que diz respeito à higiene e manutenção da saúde bucal desses idosos; através de uma revisão de literatura a respeito do tema. 


\section{MATERIAL E MÉTODO}

Trata-se de um estudo bibliográfico de abordagem quantitativa. A pesquisa abrange a busca de artigos publicados nos últimos dois anos na Scientific Electronic Library Online - SciELO sobre o tema "Condições de saúde bucal do idoso". Utilizou-se como palavras-chave: "odontologia", "idoso" e "saúde bucal".

Como critérios de inclusão, optou-se por publicações nacionais em língua portuguesa e inglesa, disponíveis completamente, justificando-se pela intenção dos autores em verificar a evolução do tema no Brasil. A escolha da base SciELO justifica-se pelo fato da mesma ser bastante difundida no que diz respeito às publicações acadêmicas. Incluiu-se exclusivamente artigos publicados entre os anos de 2014 e 2015, justificando-se pela procura da atualidade sobre o tema. Foram excluídos estudos realizados em outros países.

Ao realizar a triagem desses artigos, utilizando os critérios de inclusão, têm-se para o estudo um total de doze artigos trabalhados, sendo sete $(50 \%)$ dos artigos publicados em 2014 e cinco (50\%) dos artigos publicados em 2015.

\section{REVISÃO DA LITERATURA}

No Brasil, a transformação da antiga pirâmide populacional tem gerado grandes problemas socioeconômicos, pois uma maior quantidade de pessoas com a capacidade de desfrutar mais anos de vida nem sempre está acompanhada de saúde ${ }^{5}$.

$\mathrm{O}$ envelhecimento populacional trouxe como consequência, além do aumento da demanda por serviços de saúde, o aumento de outras modalidades de atendimento voltadas para idosos frágeis ou dependentes, como as instituições de longa permanência para idosos. Estudos mostram que a institucionalização de idosos se deve a fatores como: solidão associada à viuvez, menor oportunidade de emprego, menor suporte social e baixa renda, os quais se tornaram mais evidentes nos últimos anos $^{1}$.

Apesar disso, no Brasil não há normas específicas referentes ao cuidado da saúde bucal de idosos institucionalizados, sendo comum a ausência de protocolos de procedimentos, a falta de uma programação de atenção especializada, como também a ausência de programas de orientação e capacitação dos cuidadores de idosos. Na maioria das vezes, não possuem qualquer capacitação, o que ocasiona um desgaste tanto para o idoso quanto para estes, pois lhes falta conhecimento suficiente para orientar na manutenção de saúde bucal dos idosos ${ }^{5}$.

Existe uma grande falta de informações no tocante ao conhecimento sobre saúde bucal nessas instituições, principalmente com relação aos problemas mais comuns que ocorrem na mucosa oral, pois a maior parte dos cuidadores vê a perda dos dentes como algo inerente ao envelhecimento ${ }^{6}$.

Estudos analisaram o nível de conhecimento de alguns cuidadores que atuam em instituições asilares sobre a higiene bucal e a manutenção da saúde bucal do idoso e a falta de cooperação deste foi relatada como sendo a maior dificuldade encontrada pelos cuidadores durante a higiene bucal destes ${ }^{5}$.

Os idosos consideram a higiene bucal como uma atividade muito pessoal, e a maioria não são suscetíveis à sugestão. Portanto, os cuidados com a saúde bucal de idosos institucionalizados às vezes é complicado, sendo a maioria destes dependentes dos cuidadores, principalmente nos cuidados com sua higiene pessoal. No entanto, entre os idosos independentes poucos tiveram informações adequadas sobre saúde bucal antes de sua institucionalização ${ }^{4}$

Alguns estudos mostram que as patologias bucais encontradas em idosos se devem a certas mudanças, como o próprio o processo de envelhecimento, mudanças metabólicas, fatores nutricionais, uso de medicamentos, uso de próteses, hábitos psicopatológicos, uso de álcool e uso de tabaco ${ }^{1}$.

A saúde bucal em idosos deve ser avaliada individualmente, pois as alterações fisiológicas do envelhecimento não possuem o mesmo padrão nos diferentes organismos. Uma vez que, para muitos pacientes idosos o tratamento odontológico ainda é um desafio, em função dos prejuízos motores e cognitivos presente em grande parte destes $^{3}$

Estudos mostram que a cárie e a doença periodontal são os maiores problemas de saúde pública em odontologia, atingindo todas as idades e levando à perda dentária. Logo, a promoção de saúde do idoso garante o bem-estar, a melhoria da qualidade de vida e a autoestima desses indivíduos, pois resulta em uma mastigação e estética adequadas, possibilitando uma fácil comunicação ${ }^{6}$.

Portanto, a educação em saúde oral, além de contribuir para uma melhor estética, no idoso, preserva a função mastigatória normal e, como resultado, o estado nutritivo, pois a preservação tende a propiciar a conservação dos dentes ${ }^{7}$.

Na Tabela 1, estão algumas informações como ano de publicação, autores, título do artigo, amostra, principais resultados e conclusões dos artigos selecionados para essa revisão.

\section{DISCUSSÃO}

A população brasileira vem sofrendo alterações demográficas com o passar dos anos. Esse aumento na expectativa de vida resultou no aumento da população idosa do país. Diante do acelerado crescimento dessa população, é notória a necessidade de se proporcionar às pessoas um envelhecimento com qualidade de vida.

A saúde deve ser compreendida como um elo do organismo em funcionamento correto, com a união do bemestar físico, mental e intelectual do indivíduo. Portanto, a saúde bucal e a saúde geral se relacionam de forma a uma ocasionar sérios problemas à outra. Uma condição de saúde bucal precária poderá acarretar graves problemas à saúde geral, pois afeta o estado nutricional, comprometendo, assim, o bem-estar físico e mental, por conseguinte, diminui a qualidade de vida e o prazer da vida social do indivíduo. Do mesmo modo, o estado de saúde geral poderá ocasionar problemas bucais. Com o passar da idade, algumas dificuldades motoras se desenvolvem também, podendo comprometer a capacidade de higienização, a qual passa a ser feita por familiares ou cuidadores ${ }^{8}$.

Alguns trabalhos encontram relação no que diz respeito à saúde bucal de idosos, pois afirmam que esta população especial necessita receber uma assistência diferenciada $^{9,10}$

O envelhecimento leva a várias modificações fisiológicas em todo o organismo. A prevenção, informação e os cuidados adequados com a higiene bucal na população adulta são de extrema importância, ocorrendo isto os dentes permaneceram até a idade mais avançada.

No que diz respeito aos impactos funcionais na qualidade de vida dos idosos devido a problemas de 
Tabela 1. Descrição dos trabalhos encontrados sobre o tema em questão

\begin{tabular}{|c|c|c|c|c|}
\hline Estudo & Título & Amostra & Resultados principais & Conclusões \\
\hline Mestriner et al. $^{9}$ & $\begin{array}{l}\text { Oral health conditions and } \\
\text { quality of life of elderly } \\
\text { users of the Unified } \\
\text { National Health System }\end{array}$ & $\begin{array}{c}76 \text { idosos mentalmente } \\
\text { capacitados, independentes } \\
\text { fisicamente, ambos os sexos }\end{array}$ & $\begin{array}{c}\text { As frequências das dimensões com maior } \\
\text { impacto foram: dor }(13,16 \%) \text { e } \\
\text { desconforto psicológico }(14,47 \%) ; \text { menor } \\
\text { impacto, incapacidade social }(71 \%) \text { e } \\
\text { desvantagem social }(63 \%) \text {. }\end{array}$ & $\begin{array}{l}\text { Concluiu-se que autopercepção em } \\
\text { saúde bucal e renda dos idosos } \\
\text { expressaram impacto percebido de } \\
\text { sua saúde bucal sobre a qualidade } \\
\text { de vida. }\end{array}$ \\
\hline Lima et al. ${ }^{10}$ & $\begin{array}{l}\text { Humanização na atenção } \\
\text { básica de saúde na } \\
\text { percepção de idosos }\end{array}$ & $\begin{array}{c}\text { Estudo do tipo transversal, } \\
\text { abrangendo todas as unidades } \\
\text { básicas de saúde de um } \\
\text { município do interior do Estado } \\
\text { de São Paulo, por meio de } \\
\text { entrevistas dirigidas aos idosos } \\
\text { que frequentavam essas } \\
\text { unidades. } \\
\end{array}$ & $\begin{array}{l}54,4 \% \text { tiveram liberdade para tomar } \\
\text { decisões sobre sua saúde ou tratamento; } \\
67,6 \% \text { não obtiveram informações sobre } \\
\text { outros tipos de tratamentos ou exames e } \\
79,2 \% \text { não tiveram oportunidade para } \\
\text { esclarecimentos quanto às dúvidas sobre } \\
\text { o tratamento. }\end{array}$ & $\begin{array}{l}\text { Sugere-se, portanto, mudanças nos } \\
\text { serviços de atenção básica à saúde } \\
\text { prestados, principalmente quando se } \\
\text { trata de idosos, uma população } \\
\text { especial que necessita receber uma } \\
\text { assistência diferenciada. }\end{array}$ \\
\hline $\begin{array}{l}\text { Magalhães Junior } \\
\text { et al. }^{11}\end{array}$ & $\begin{array}{l}\text { Caracterização da } \\
\text { pressão da língua em } \\
\text { idosos. }\end{array}$ & $\begin{array}{c}45 \text { idosos, de } 61 \text { a } 96 \text { anos, } \\
\text { sendo } 34(75,6 \%) \text { do gênero } \\
\text { feminino e } 11 \text { ( } 24,4 \%) \text { do } \\
\text { masculino, sem distúrbios } \\
\text { neurológicos e cognitivos, sem } \\
\text { histórico de câncer de cabeça e } \\
\text { pescoço e de procedimento } \\
\text { radioterápico. }\end{array}$ & $\begin{array}{l}\text { Os idosos apresentaram a menor média } \\
\text { de pico pressórico e inferior ao padrão de } \\
\text { normalidade. Houve diferença entre a } \\
\text { média de pico pressórico e uso de } \\
\text { próteses, tensão de língua e mobilidade } \\
\text { de língua no estalo. }\end{array}$ & $\begin{array}{l}\text { Houve diminuição moderada do pico } \\
\text { pressórico da língua com o aumento } \\
\text { da idade e redução pressórica com o } \\
\text { decréscimo da tensão da língua e de } \\
\text { sua mobilidade no estalo. }\end{array}$ \\
\hline Morais et al. ${ }^{12}$ & $\begin{array}{l}\text { Factors related to dry } \\
\text { mouth and low salivary } \\
\text { flow rates in diabetic } \\
\text { elderly: a systematic } \\
\text { literature review. }\end{array}$ & $\begin{array}{l}\text { Cinco artigos foram } \\
\text { selecionados, sendo quatro } \\
\text { estudos transversais e um } \\
\text { longitudinal. }\end{array}$ & $\begin{array}{l}\text { Alguns trabalhos encontraram relação } \\
\text { entre o mau controle glicêmico e o } \\
\text { surgimento de hipossalivação. Outros } \\
\text { encontraram que a xerostomia foi mais } \\
\text { frequente em pacientes que trabalhavam } \\
\text { em ambientes não domésticos e em } \\
\text { mulheres. }\end{array}$ & $\begin{array}{l}\text { Conclusões definitivas não puderam } \\
\text { ser retiradas com base na análise } \\
\text { dos trabalhos selecionados. }\end{array}$ \\
\hline Batista et al. ${ }^{13}$ & $\begin{array}{c}\text { Relationship between } \\
\text { lower-limb muscle strength } \\
\text { and functional } \\
\text { independence among } \\
\text { elderly people according } \\
\text { to frailty criteria: a cross- } \\
\text { sectional study } \\
\end{array}$ & $\begin{array}{l}\text { Estudo realizado entre outubro } \\
\text { de } 2005 \text { e outubro de } 2007 \text { com } \\
150 \text { idosos ambulatoriais de } \\
\text { ambos os sexos, com condições } \\
\text { cognitivas suficientes para } \\
\text { comunicação oral. }\end{array}$ & $\begin{array}{l}\text { O tempo médio no teste de levantar e } \\
\text { sentar da cadeira foi de } 21,7 \text { segundos, a } \\
\text { pontuação média da MIFm de } 82,2 \text { e da } \\
\text { AIVDt de } 21,2 ; 44,7 \% \text { dos sujeitos } \\
\text { apresentaram } 1-2 \text { critérios de fragilidade } \\
\text { e } 55,3 \%>3 \text { critérios. }\end{array}$ & $\begin{array}{l}\text { Os idosos com um ou dois critérios } \\
\text { de fragilidade apresentaram melhor } \\
\text { independência em todos os escores } \\
\text { do teste de levantar e sentar da } \\
\text { cadeira. Os sujeitos com maior FM } \\
\text { de MMII apresentaram melhor } \\
\text { independência funcional. }\end{array}$ \\
\hline Martins et al. ${ }^{14}$ & $\begin{array}{c}\text { Associação entre } \\
\text { impactos funcionais e } \\
\text { psicossociais das } \\
\text { desordens bucais e } \\
\text { qualidade de vida entre } \\
\text { idosos } \\
\end{array}$ & $\begin{array}{c}\text { Dos } 800 \text { convidados, } \\
\text { participaram } 736 \text { idosos (TR }= \\
\text { 92\%), com a média de idade de } \\
67,77 \text { anos }\end{array}$ & $\begin{array}{c}\text { A maioria não apresentou impacto, a } \\
\text { partir da medida da prevalência do OHIP. } \\
\text { A dimensão limitação funcional do OHIP } \\
\text { foi associada ao domínio físico do SF12, } \\
\text { independentemente de outras variáveis } \\
\text { investigadas. }\end{array}$ & $\begin{array}{l}\text { Conclui-se que alguns impactos das } \\
\text { desordens bucais estiveram } \\
\text { associados a uma qualidade de vida } \\
\text { insatisfatória em seus domínios físico } \\
\text { e mental. }\end{array}$ \\
\hline Ferreira et al. ${ }^{15}$ & $\begin{array}{c}\text { O idoso com } \\
\text { comprometimento } \\
\text { cognitivo apresenta pior } \\
\text { condição de saúde bucal? }\end{array}$ & $\begin{array}{l}\text { Foram entrevistados e } \\
\text { examinados } 736 \text { idosos. }\end{array}$ & $\begin{array}{l}\text { Os com comprometimento cognitivo } \\
\text { apresentaram maiores médias de CPOD, } \\
\text { COR e menores médias de sextantes } \\
\text { sadios do CPI, prevalências menores de } \\
\text { sextantes sem placa/cálculo e de uso de } \\
\text { prótese; maiores prevalências de } \\
\text { edentulismo e necessidade de próteses. }\end{array}$ & $\begin{array}{l}\text { Idosos com comprometimento } \\
\text { cognitivo apresentaram pior saúde } \\
\text { bucal }\end{array}$ \\
\hline Yoshida et al. ${ }^{16}$ & $\begin{array}{l}\text { A influência da função } \\
\text { mastigatória na deglutição } \\
\text { orofaríngea em idosos } \\
\text { saudáveis. }\end{array}$ & $\begin{array}{l}\text { Foram analisados os dados de } \\
47 \text { idosos saudáveis - } 29 \\
\text { mulheres e } 18 \text { homens }\end{array}$ & $\begin{array}{l}\text { A maioria dos indivíduos apresentou tipo } \\
\text { mastigatório bilateral (62\%), formação do } \\
\text { bolo alimentar adequada (75\%) e cerca } \\
\text { de metade ( } 47 \%) \text {, quadro de disfagia } \\
\text { orofaríngea de grau moderado. }\end{array}$ & $\begin{array}{l}\text { Para os idosos deste estudo, o } \\
\text { tempo mastigatório influenciou o } \\
\text { grau de disfunção da deglutição, não } \\
\text { tendo sido encontrada relação para o } \\
\text { tipo mastigatório e formação do bolo } \\
\text { alimentar quanto aos achados da } \\
\text { deglutição orofaríngea. }\end{array}$ \\
\hline Costa et al. ${ }^{17}$ & $\begin{array}{l}\text { Diadococinesia oral e } \\
\text { função mastigatória em } \\
\text { idosos saudáveis. }\end{array}$ & $\begin{array}{c}\text { Análise de prontuários e } \\
\text { arquivos de imagens de } 35 \\
\text { idosos saudáveis, entre } 60 \text { e } 74 \\
\text { anos de idade. }\end{array}$ & $\begin{array}{l}\text { A análise estatística demonstrou } \\
\text { correlação entre o tempo mastigatório e } \\
\text { os parâmetros coeficiente de variação do } \\
\text { pico da intensidade da sílaba "ta" e } \\
\text { perturbações do período da sílaba "ka", } \\
\text { indicando instabilidade da DDC. }\end{array}$ & $\begin{array}{l}\text { A DDC oral correlacionou-se com o } \\
\text { tempo mastigatório em idosos } \\
\text { saudáveis, evidenciando que, quanto } \\
\text { maior a instabilidade na repetição } \\
\text { dos movimentos de ponta e dorso de } \\
\text { língua durante a produção } \\
\text { articulatória, maior o tempo } \\
\text { necessário para a preparação do } \\
\text { alimento para a deglutição. }\end{array}$ \\
\hline Martins et al. ${ }^{18}$ & $\begin{array}{l}\text { Access to information on } \\
\text { how to avoid oral health } \\
\text { problems among elderly } \\
\text { persons registered with } \\
\text { the Family Health } \\
\text { Strategy. }\end{array}$ & 490 participantes & $\begin{array}{l}53,4 \% \text { relataram o acesso a informações } \\
\text { sobre como evitar problemas bucais. } \\
\text { Esse acesso foi maior entre os idosos } \\
\text { que residiam em domicílio cadastrado na } \\
\text { ESF, tinham maior escolaridade, } \\
\text { utilizaram os serviços odontológicos por } \\
\text { rotina, não relataram hábito tabagista } \\
\text { atual ou passado, não possuíam doença } \\
\text { crônica e não tiveram impacto social das } \\
\text { suas condições de saúde bucal. }\end{array}$ & $\begin{array}{c}\text { Conclui-se que a maioria dos idosos } \\
\text { teve acesso a informações sobre } \\
\text { como evitar problemas bucais, sendo } \\
\text { o acesso maior entre aqueles } \\
\text { cadastrados na Estratégia de Saúde } \\
\text { da Família. }\end{array}$ \\
\hline Rigo et al. ${ }^{19}$ & $\begin{array}{l}\text { Satisfação com a vida, } \\
\text { experiência odontológica } \\
\text { e autopercepção da saúde } \\
\text { bucal entre idosos. }\end{array}$ & $\begin{array}{l}326 \text { idosos acima de } 60 \text { anos de } \\
\text { idade, moradores de um } \\
\text { município do norte do Rio } \\
\text { Grande do Sul, Brasil. }\end{array}$ & $\begin{array}{l}\text { Os resultados mostraram que idosos com } \\
\text { maiores níveis de satisfação com a vida } \\
\text { apresentaram uma melhor percepção de } \\
\text { sua própria saúde bucal, bem como } \\
\text { possuem uma melhor imagem percebida } \\
\text { dos cirurgiões-dentistas e sentem menos } \\
\text { ansiedade em relação à experiência com } \\
\text { o dentista. }\end{array}$ & $\begin{array}{l}\text { Tanto a autopercepção que os } \\
\text { idosos possuem da saúde bucal } \\
\text { quanto a experiência com cirurgiões- } \\
\text { dentistas possuem uma associação } \\
\text { com a satisfação com a vida. }\end{array}$ \\
\hline Martins et al. ${ }^{20}$ & $\begin{array}{l}\text { Prevalência de autoexame } \\
\text { bucal é maior entre idosos } \\
\text { assistidos no Sistema } \\
\text { Único de Saúde: inquérito } \\
\text { domiciliar }\end{array}$ & $\begin{array}{l}740 \text { avaliados, atenderam aos } \\
\text { critérios de inclusão } 492 \text { idosos } \\
\text { e, destes, } 101(22,4 \%) \\
\text { relataram a prática do } \\
\text { autoexame bucal }\end{array}$ & $\begin{array}{l}101(22,4 \%) \text { relataram a prática do } \\
\text { autoexame bucal. Esta prática foi maior } \\
\text { entre idosos usuários dos serviços } \\
\text { odontológicos prestados no SUS, entre } \\
\text { aqueles com maior renda per capita, os }\end{array}$ & $\begin{array}{l}\text { O estímulo à adesão a este } \\
\text { autocuidado deve ser considerado } \\
\text { nas políticas de saúde do idoso } \\
\text { vigentes, especialmente entre } \\
\text { usuários de serviços particulares, }\end{array}$ \\
\hline
\end{tabular}


desordens bucais; observou-se que essas desordens estariam associadas a uma qualidade de vida insatisfatória, tanto na parte física quanto mental ${ }^{13,14,15,18}$.

$\mathrm{O}$ atendimento odontológico domiciliar para o idoso parcial e totalmente dependente caracteriza-se por um conjunto de ações preventivas e de mínima intervenção com a finalidade de promover a saúde bucal e orientar familiares e cuidadores. Estes devem estar preparados também para as condições de manejo psicológico, as quais podem levar a aceitações negativas por parte do idoso. Muitos cuidadores apresentam dificuldade e insegurança, necessitando assim, de mais instruções quanto ao cuidado de higiene bucal do idoso $^{2}$.

No Brasil não há normas específicas para o cuidado da saúde bucal de idosos institucionalizados, o que reforça a necessidade de uma maior atenção para esta população, que muitas vezes tem seus cuidados negligenciados.

\section{CONSIDERAÇÕES FINAIS}

Pode-se concluir com esse trabalho que com os avanços tecnológicos e com o aumento da expectativa de vida, têm-se idosos mais saudáveis, com baixas patologias, mas essas ainda prejudiciais para a saúde desses idosos se não tratada e diagnosticada precocemente.

Observa-se ainda uma maior necessidade de tratamento odontológico voltado para essa população, devido às patologias oriundas do próprio envelhecimento. Tal conhecimento permite aos profissionais de saúde a trabalharem nas ações de promoção de saúde e prevenção dos agravos.

Considerada como sendo de primordial importância fisiológica e metabólica, a cavidade bucal passa a sofrer com a chegada da idade. Em idosos institucionalizados essa situação é ainda mais crítica em relação à população idosa em geral, devido a associação de diversas doenças sistêmicas, além de precários cuidados de saúde bucal.

\section{REFERÊNCIAS}

1. Paula BG, Almeida MRB, Alves JFCS. Alterações bucais de idosos institucionalizados - Revisão de literatura. Rev Odontol Univ Cid São Paulo. 2014; 26(3):219-26.

2. Rocha DA, Miranda AF. Atendimento odontológico domiciliar aos idosos: uma necessidade na prática multidisciplinar em saúde: revisão de literatura. Rev Bras Geriatr Gerontol. 2013; 16(1):181-9.

3. Oliveira JAG, Oliveira LLN, Cunha VPP. Considerações no tratamento em Odontogeriatria: relato de caso clínico. Arch Health Invest. 2014; 3(6):1-5.

4. Silva BLA, Bonini JA, Bringel FA. Condição de saúde bucal de idosos institucionalizados em Araguaína/TO. Braz J Periodontol. 2015; 25(1):7-13.

5. Alves VM, Tomo S, Simonato LE, Pereira AM, Boer NP, Cunha-Correia AS, Lima DP. Avaliação do conhecimento de cuidadores sobre a higiene bucal de pacientes idosos institucionalizados. Arch Health Invest. 2014; 3(5):9-15.

6. Monteiro TAS, Monteiro DCC, Monteiro MSS, Monteiro LSS, Lima LAA, Queiroz AAFLN Cuidadores de idosos e atitudes frente à saúde bucal: uma revisão integrativa. Rev Enferm UFPI. 2013; 2(spe):102-7.

7. Chagas AM, Rocha ED. Aspectos fisiológicos do envelhecimento e contribuição da Odontologia na saúde do idoso. Rev Bras Odontol. 2012; 69(1):94-6.
8. Rovida TAS, Peruchini LFD, Moimaz SAS, Garbin CAS. O conceito de saúde gera e bucal na visão dos cuidadores de idosos. Odontol Clín-Cient. 2013; 12(1):43-6.

9. Mestriner SF, Almeida ASQ, Mesquita LP, Bulgarelli AF, Mestriner Junior W. Condições de saúde bucal e qualidade de vida de idosos usuários do Sistema Único de Saúde. RGO. 2014; 62(4):389-94.

10. Lima TJV, Arcieri RM, Garbin CAS, Moimaz SAS, Saliba O. Humanização na atenção básica de saúde na percepção de idosos. Saude Soc.2014; 23(1):265-76.

11. Magalhães Junior HV, Tavares JC, Magalhães AAB, Galvão HC, Ferreira MAF. Caracterização da pressão da língua em idosos. Audiol Commun Res. 2014; 19(4):375-9.

12. Morais EF, Macedo RAP, Lira JAS, Lima KC, Borges BCD. Factors related to dry mouth and low salivary flow rates in diabetic elderly: a systematic literature review. Rev Bras Geriatr Gerontol. 2014; 17(2):417-23.

13. Batista FS, Gomes GAO, D'elboux MJ, Cintra FA, Neri $\mathrm{AL}$, Guariento ME, et al. Relação entre força muscular de membros inferiores e independência funcional de idosos segundo critérios de fragilidade: um estudo transversal. São Paulo Med J. 2014; 132(5):282-9.

14. Martins AMEBL, Jones KM, Souza JGS, Pordeus IA. Associação entre impactos funcionais e psicossociais das desordens bucais e qualidade de vida entre idosos. Ciênc saúde coletiva. 2014; 19(8):3461-78.

15. Ferreira RC, Vargas AMD, Fernandes NCN, Souza JGS, Sá MAB, Oliveira LFB, et al. O idoso com comprometimento cognitivo apresenta pior condição de saúde bucal? Ciênc saúde coletiva. 2014; 19(8):3417-28.

16. Yoshida FS, Mituuti CT, Totta T, Berretin-Felix G. A influência da função mastigatória na deglutição orofaríngea em idosos saudáveis. Audiol Commun Res. 2015; 20(2):161-6.

17. Costa DR, Totta T, Silva-Arone MMA, Brasolotto AG, Berretin-Felix G. Diadococinesia oral e função mastigatória em idosos saudáveis. Audiol Commun Res. 2015; 20(3):191-7.

18. Martins AMEBL, Haikal DS, Souza JGS, Sá MAB, Ferreira EF, Pordeus IA. Access to information on how to avoid oral health problems among elderly persons registered with the Family Health Strategy. Rev Bras Geriatr Gerontol. 2015; 18(4):855-9.

19. Rigo L, Basso K, Pauli J, Cericato GO, Paranhos LR, Garbin RR. Satisfação com a vida, experiência odontológica e autopercepção da saúde bucal entre idosos. Ciênc saúde coletiva. 2015; 20(12):3681-8.

20. Martins AMEBL, Souza JGS, Haikal DS, Paula AMB, Ferreira EF, Pordeus IA. Prevalência de autoexame bucal é maior entre idosos assistidos no Sistema Único de Saúde: inquérito domiciliar. Ciênc saúde coletiva. 2015; 20(4):1085-98, 2015. 
Arch Health Invest (2017) 6(3): 120-124

\section{CONFLITO DE INTERESSES}

Os autores declaram não haver conflitos de interesse.

\section{AUTOR PARA CORRESPONDÊNCIA}

Maria Helena Chaves de Vasconcelos Catão

mhelenact@zipmail.com.br

Submetido em 27/12/2016

Aceito em 26/01/2017 